\title{
A Python-Based Interface Design for Electric Power System Education
}

\author{
Yousef Marwan Alkhanafseh and Tahir Cetin Akinci*
}

\begin{abstract}
Python, which is widely used today, is one of the open-source programming languages. In the Python program, its interface is done through the PyQt5 library. With PyQt5, it is possible to develop object-based software that takes up less space on the disk and runs faster. In this study, an educational calculation and analysis program (ITUPSA) was designed for the Electric power systems course using the Python interface. Electric power systems are one of the fundamental courses in electrical engineering. This main course is taught in three subcourse groups as energy transmission-distribution, energy flow and energy economy. The energy transmission-distribution course package constitutes the first and most comprehensive part of the electric power systems group. In the energy transmission-distribution, the creation of the transmission line model and the calculation of the line parameters form an important part of the training. This section contains very detailed mathematical calculations and it is very important that students get the correct result as a result of these calculations. This can be possible with a lot of practice. The interface design was made by students and faculty to teach practical examples correctly. This developed (ITUPSA) python-based interface program is very suitable for calculation and analysis exercises in both undergraduate and graduate courses in the Electrical Engineering department. The purpose of this study is to create a python-based interface in order to solve the problems related to these lines, to make the types of short, medium and long power transmission lines correct and understandable. In the study, after selecting the type of energy transmission line and all parameters related to the line, the necessary mathematical analysis can be made and also the analysis results can be plotted. The analysis program is available in two languages, English and Turkish.
\end{abstract}

Index Terms: Electric Power Transmission, Electric Power System Education, Python Gui, PyQt5.

\section{INTRODUCTION}

$\mathrm{T}$ he history of the electric power system began in 1881 with the generation and distribution of electrical energy from a water wheel in England. In 1882, Edison built a steam-powered power plant in New York and carried the energy is produced to a distance of 800 meters [1]. In the same years, by using Gaulard and Gibbs transformers in London, they carried the energy to $40 \mathrm{~km}$ distances $[1,2]$.

Manuscript received July 2, 2020; revised September 10, 2020; accepted April 15, 2021.

Yousef Marwan Alkhanafseh is with Department of Electrical Engineering, Istanbul Technical University, Istanbul, Turkey (e-mail: alkhanafseh@itu.edu.tr).

Tahir Cetin Akinci is with Department of Electrical Engineering, Istanbul Technical University, Istanbul, Turkey (e-mail: akincitc@itu.edu.tr).

*Corresponding author.
In today's sense, the technique of using energy by transforming would only be possible with the development of transformers.

The development of transformers and the transmission of power lines to the present were realized with the contributions of Ganz, Blathy, Kennedy, Barrington, Westinghouse, Edison and Tesla [2-6]. In 1888, cities were enlightened using energy transmission lines [4-7]. When the three-phase alternating current system was found in 1895 , general developments regarding the electric power system were completed [8-10]. In the following years, the development of electrical power system components and intermediate elements was to ensure a better quality transmission of energy [11-13]. Electric power systems captured their next biggest development with renewable energy sources and smart grid approach [14]. Both the smart grid and renewable energy technologies are developing technology in electrical power systems today. This technology has gained momentum with the spread of innovations in the field of information and communication technology (IT) in the field of power engineering [15].

In recent years, economic regimes in electricity energy consumption have increased the tendency to train more experienced electric power engineers in the energy markets. Increasing energy demand in the electricity industry, ageing of power transmission and distribution lines, energy security targets direct countries to smart grid technologies [16]. The problem of qualified personnel experienced in the widespread use of smart grid technologies, distributed and renewable energy sources in electrical power engineering create important training and demand need for the sector.

The difficulty of education keeps the demand for electrical power engineering programs low in many developed countries [17]. The most important reason for this is the decrease in industrial support to electrical power engineering programs and the difficult training [18]. At the same time, the introduction of new technologies in electrical energy systems, the liberalization of the electricity markets, the support of the use of renewable energy sources by the governments complicates the e. nergy supply-demand energy sector [18]. All this mess creates the need to increase technical infrastructure requirements for students graduating from electrical power engineering. In addition to the classical electrical engineering education, it also led students to take lessons including information technology and artificial intelligence methods for the smart grid and energy market economy [19]. Despite all these demands and the changes brought by technological developments, electric power education renews itself with new reforms and finds new ways to prepare its students for the markets. [20-22]. In this sense, electrical power engineering education is constantly changing in order to train students with the quality 
demanded by the markets [23]. Many studies have proven that the use of modelling and simulation software that provides feedback during the education of students facilitates learning [24].

Planning and development of smart grids are only possible with a very good understanding of the training of electrical power systems [18]. This requirement can be achieved by developing an object-based interface program and applying it to lessons that require a more practical application. In recent years, many object-oriented commercial programs have been developed for the analysis of electrical power systems. Most analysis programs developed are designed to be used by experienced engineers according to industry needs [18]. However, these programs provide a great convenience for project engineers by using all of the advanced analysis methods together according to the needs of the industry. Commercial uses provide enormous convenience as the majority of commercial programs also contain many electrical components available on the market as codes. However, these commercial programs are sold under license for high prices for commercial enterprises. In addition, these commercial programs companies offer a series of training seminars to post-sales engineer users, ensuring that their programs are used efficiently. Although commercial programs are also used by trained experienced engineers, it raises the need to produce new versions of the program to keep up with the developing technology. These new versions give rise to commercial version upgrade fees and re-orientation training. These commercial programs are constantly changing and developing to meet all the needs of developing technology by very large software companies. These programs are not the software that electrical engineering students can use during their education with their current knowledge. Electrical engineering students need free software that can quickly apply and practice many examples [26-28].

Simulation and analysis programs in electrical power systems are divided into two main groups for commercial and educational purposes. There are several large and medium-sized commercial programs on the market. These commercial programs are designed to perform detailed modelling and simulation of systems. It is suitable for commercial projects. However, these programs are inadequate for research and educational use. The most important reason for this is that these programs are made for professional engineers. It requires a high degree of knowledge for students, their use also requires training, and the results are suitable for commercial projects. One of the most important disadvantages of these commercial programs not being used in education is that they do not allow changing algorithms or adding new models. The most anticipated expectation from programs designed for educational purposes is the flexibility to carry out research and education objectives, and their ability to perform simple and basic calculations. Although there are several programs in this field, they are not suitable for individual use due to their high costs [26-30]. It is known that the electric power systems can be thought of as the group power transmission lines carrying energy from the power generation facilities to the end-user. There are three different types of electrical power transmission networks, namely short, medium and long lines, in the transmission of electrical energy. Depending on the type, the length, cable feature and diameter of these networks play an important role in the transmission and distribution of electrical energy to different distances.

In this study, educational software for electrical power engineering was developed using Python PyQt5 interface library. With the software, electrical power engineering students were given the opportunity to practice by applying the problems in power systems. The software has been developed in Istanbul Technical University Electric Power Systems department and has been tested for the students in this department for practical use. In this study, it is focused only on the design of the software. This study has features to be used especially by integrating with renewable energy sources.

\section{POWER SYSTEM COURSE AND APPLICATIONS}

\subsection{Power System Course}

The Power Systems Course (PSC) deals with models, calculations and analyzes related to the analysis of the fundamental electrical power system. Models and methods used in Electric Power systems are quite general and can be applied to electric power systems of variable scales. These scales can be combined with national and international electricity transmission networks, such as a network. In general terms, Electric Power Systems can be divided into several sub-courses or collected in a single course in general. In this undergraduate course, numerical calculation techniques, modelling, analysis and control techniques are taught. In the first stage, general information about the general structure, equipment and parameters of electrical power systems is given. In the second stage, AC transmission systems and the installation of these systems are explained. In the third stage, the behaviour of energy transmission and distribution systems under loads is explained by numerical calculation methods. Here, energy losses, state estimation and load flow are given. Advanced topics of electrical power systems can also be taught as a course. These courses can sometimes be moved to the master program. These advanced topics include energy economy, energy market, asymmetrical conditions of power systems, error analysis, stability, optimization and control of energy systems.

A student who has successfully taken the fundamental of electrical power systems course can take all other power system courses and specialize in this field. Students who take the course of the fundamentals of the electric power system learn the following topics;

- Transmission line parameters;

- Inductance and capacitance,

- Short, medium and longline models and currentvoltage relationships

- Electrical characteristics of transformers and generators,

- Per-Unit system,

- Modelling and Analysis of Power Systems.

The Energy Transmission Lines Engineering course content, which is a continuation of this course, consists of the following general headings.

- Calculation of power line materials against strength and overvoltages. Protection angle calculation and chain curve equation in high voltage lines.

- Deflection calculation and deflection methods. 
- Wire drawing calculations and additional loads on energy transport lines.

- Openings and calculations between poles.

- Calculation of economic and critical gaps.

- Poles and lattice mast calculations. Concrete poles and their calculations.

- Grounding resistance in overhead lines.

- Introduction to energy transmission stability.

- Integration into renewable energy systems.

\subsection{Undergraduate Student Work}

In electrical power systems course, the course is divided into two parts: theoretical and practical. In the theoretical part, students form groups and solve numerical problems given in the course. Students 'exam success is closely related to students' understanding of numerical problems very well. The results of the numerical problems solved must be tested using the software. Afterwards, the groups discuss the different examples and complete the theoretical part of the lesson. There are fundamental typical problems to be solved in each lesson. After these problems are solved with classical methods, the results of the university are tested with software. For this software, students are practised by the research assistants before the lesson.

In general, numerical calculations of electrical power systems are solved with electrical circuit software after mathematical models are simplified. However, as the lessons progress, the parameters used in the transmission and distribution lines include special parameters and components. In this case, if the universities do not have special software, the subject is skipped by informing about the software that the solutions can be made. Scientists working in this field know very well the necessity of teaching this area of the course, especially by making student-centered software. The software made in this study is designed to develop the applications that students will do after the theoretical part.

Laboratory practices of students are carried out with systems with real data collection system with transformers, motors and lines. Figs. 1-3 shows the Energy-Transmission and distribution Laboratory, which ITU Electrical engineering students use under real conditions.

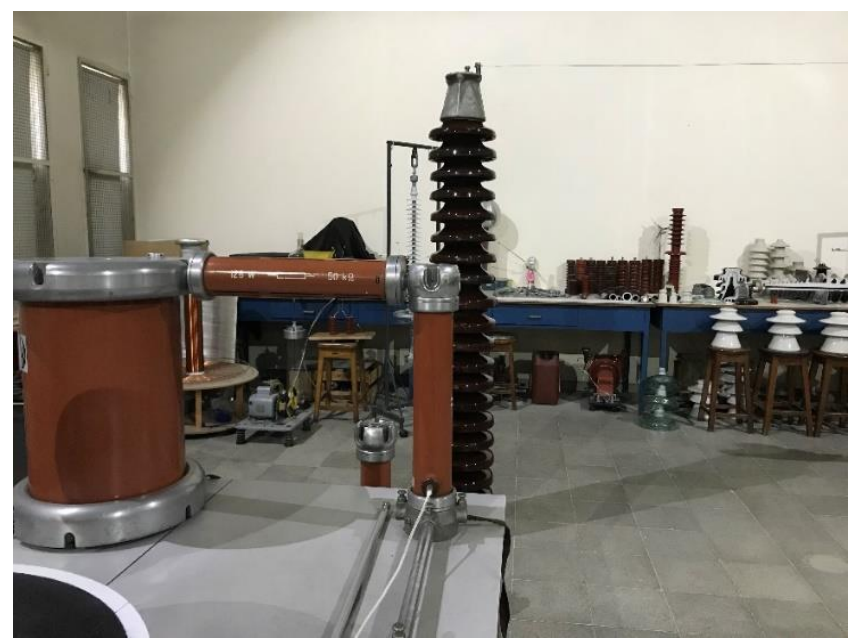

Fig. 1. ITU Energy Transmission Systems Lab2.

However, it may not always be economical to renew laboratory equipment with developing technology. It is very important for students to work in laboratories equipped with real equipment in order to gain practical experience and recognize electrical power system components. It is not economical to renew these laboratories, which are equipped with highly expensive electrical power systems, according to developing technological developments.

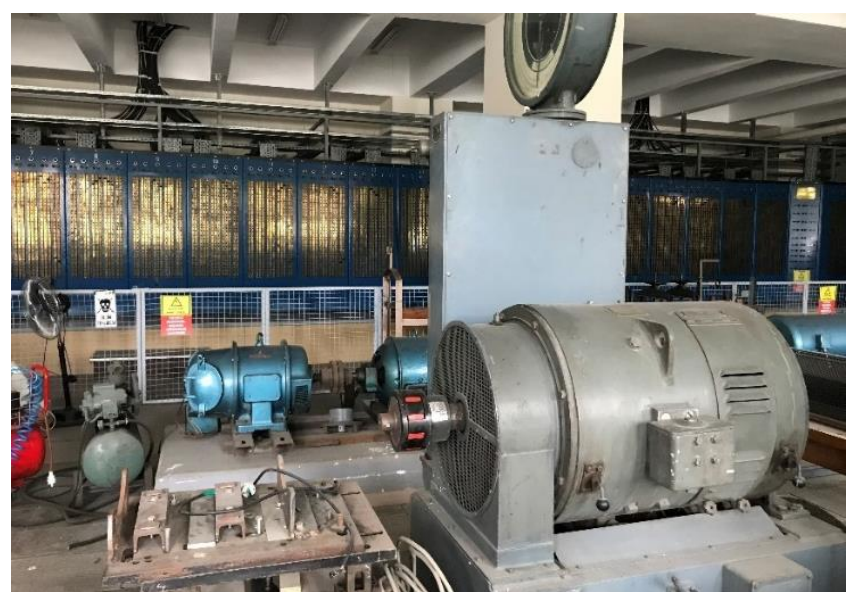

Fig. 2. ITU Energy Distribution Systems Lab.

It is also necessary to take the necessary safety precautions during the experiment in real conditions. For this reason, these laboratories should be in terms of learning fundamental concepts, but courses should be supported by technological software. In Fig. 3, ITU Power Systems Lab. Analogue Energy Transmission Simulator is shown. Here, analogue energy transmission simulator $\mathrm{Pi}$ and $\mathrm{T}$ model modules can be seen.

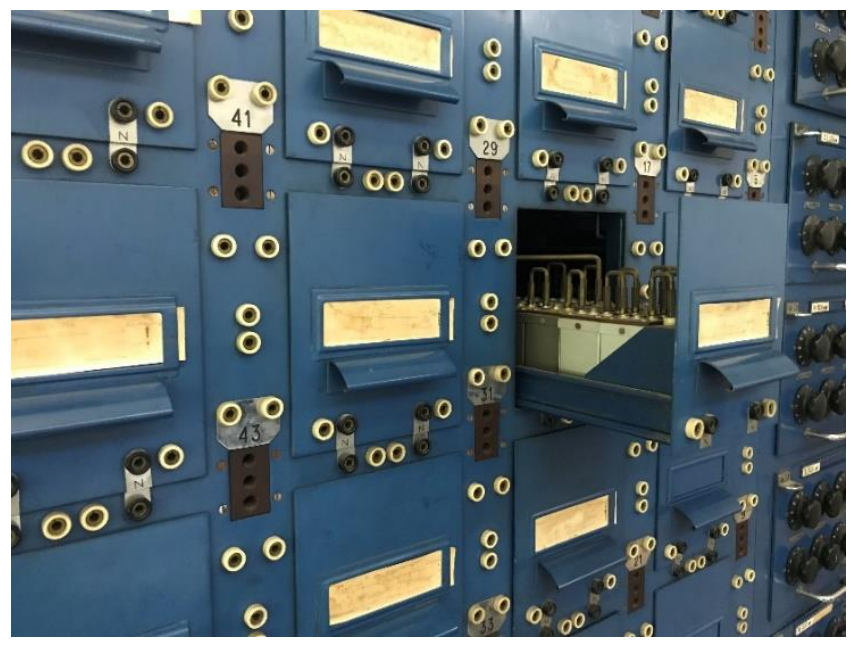

Fig. 3. ITU Power Systems Lab. Analog Energy Transmission Simulator, $\mathrm{Pi}$ and $\mathrm{T}$ model modules

\section{ITUPSA SOFTWARE IMPLEMENTATION FOR POWER SYSTEM LABORATORY}

With the development of object-oriented programming and visual programming, the power system programs used in the past have been replaced by new object-oriented power system analysis programs. These programs are easy-to-use software that can give problem results and analysis results on the same page with the graphic interface unit. With this software, data entry in standard transmission line diagrams 
can be made easily with mouse or keyboard. The software, which can give analysis results very fast, is $200 \mathrm{MB}$ in size with all its subfiles.

ITUPSA is a software developed to practice students for planning, modelling and analysis of electrical power systems. The software was developed using the Pythonbased PyQt5 interface. Due to the Python interface, it is not commercial software, and since the program is open-code, it can be developed by people who are interested in power systems in later versions. Starting his education life in 1173 , Istanbul Technical University (ITU) started his electrical engineering education in 1926. The free version of ITUPSA is used by ITU electrical engineering students. In the researches, it has been concluded that the students have great benefits in terms of controlling the solutions of their theoretical problems and that they facilitate learning. The program focuses on analyzing energy transmission and distribution line problems. It has two language options and an easy-to-use interface. The graphic interface is quite powerful.

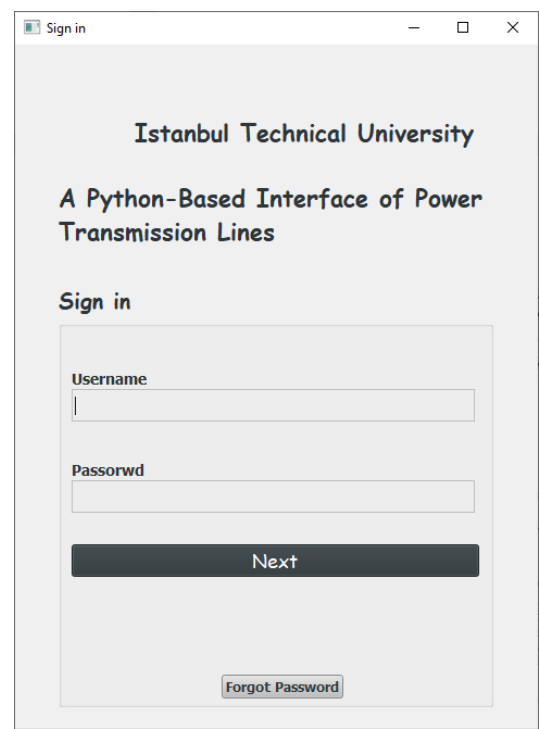

Fig. 4. ITUPSA Login Screen

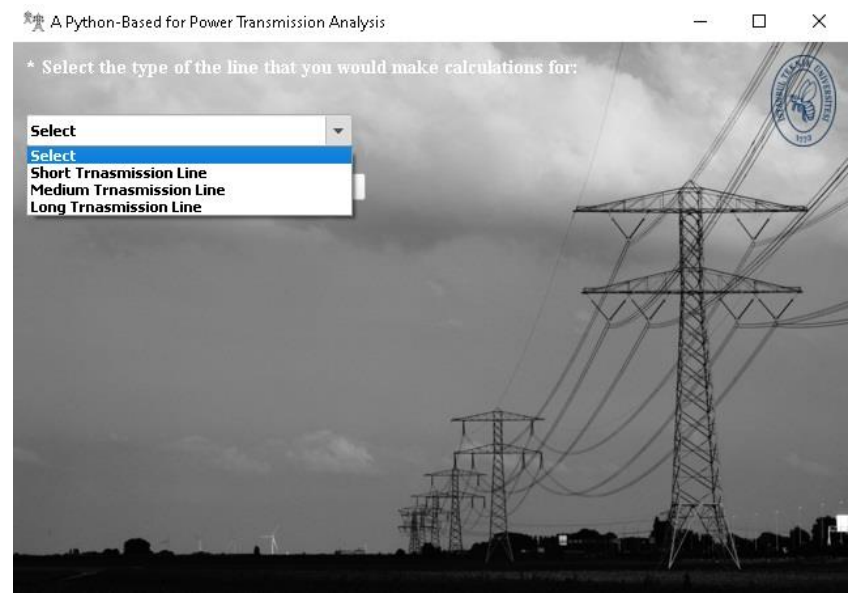

Fig. 5. Selection screen for Short, Medium and Long transmission line

As shown in Fig. 4, students can $\log$ in directly to the program using the password given by the instructor, using the user name and password. The transmission line options of ITUPSA program are shown in Fig. 5 and its interface is shown in Fig. 6.

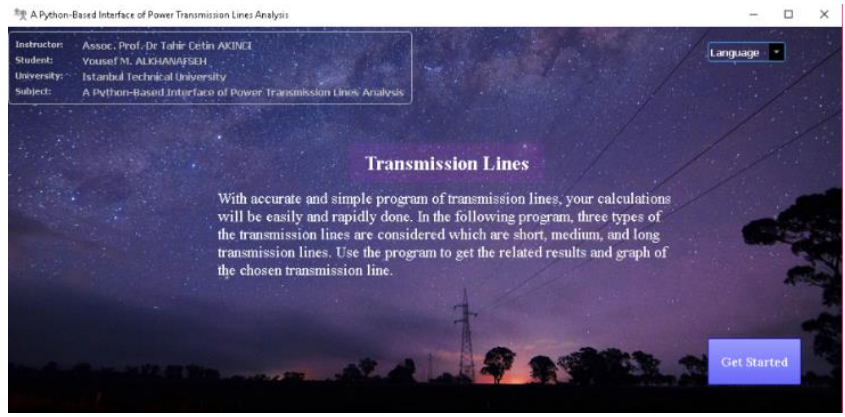

Fig. 6. ITUPSA interface

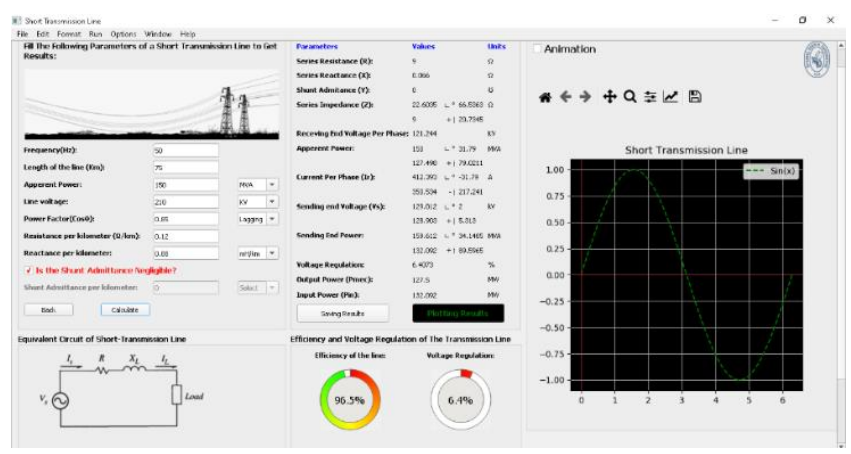

Fig. 7. ITUPSA Short Transmission Parameters and Transmission Line Analysis.

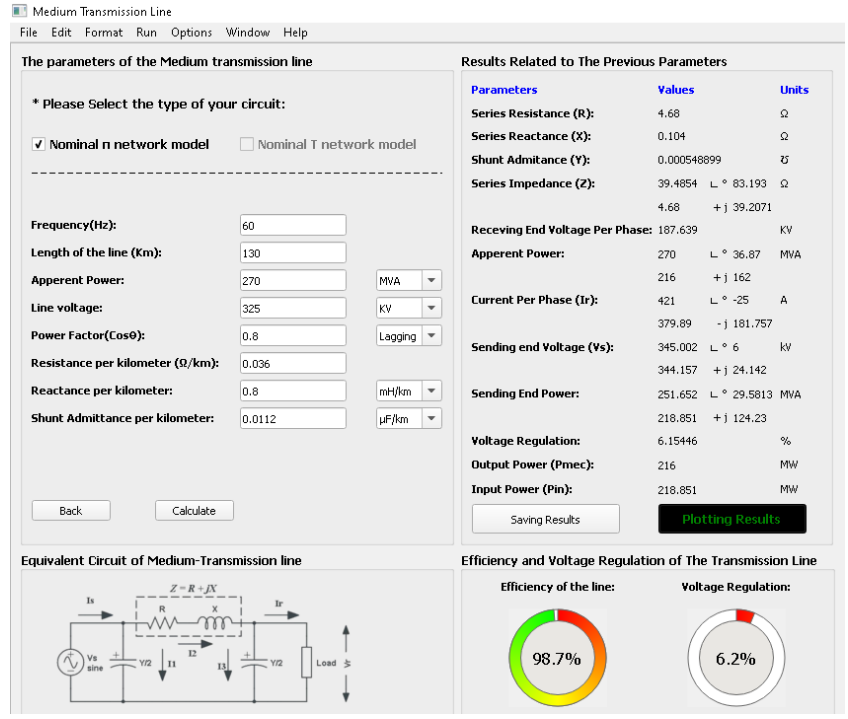

Fig. 8. ITUPSA Middle Transmission Line Parameter Interface

As a result of the parameters entered, the calculated results and analysis of the short transmission line are given in Fig. 7. When the required parameters are entered for the medium length transmission line, Serial resistance, series reactance, serial admittance, series impedance, apparent power, Current per-phase voltage regulation values are calculated (Fig. 8).

In addition, the efficiency of the line and voltage regulation values are calculated and displayed on the interface. 


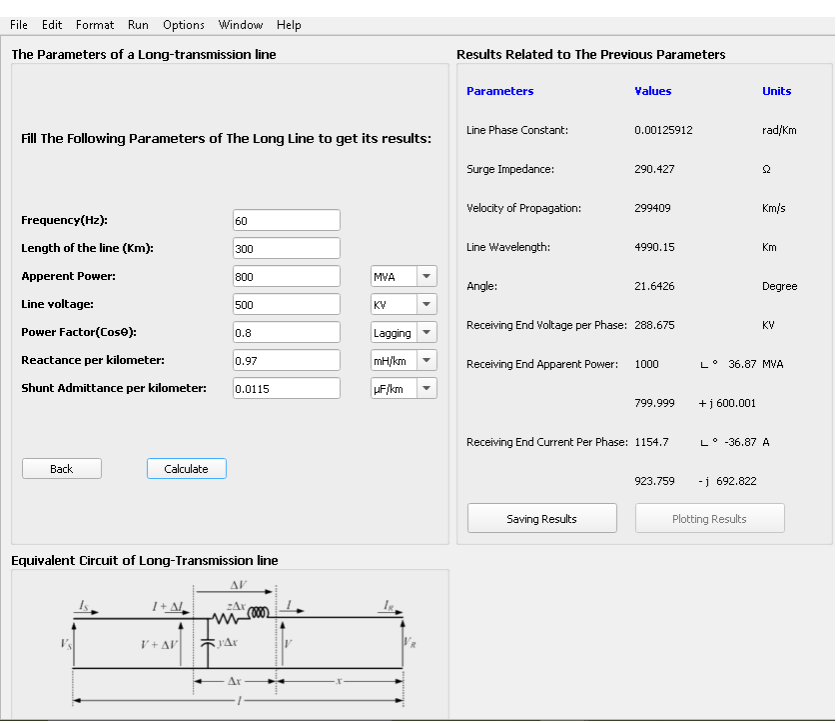

Fig. 9. ITUPSA Middle Transmission Line Parameter Interface

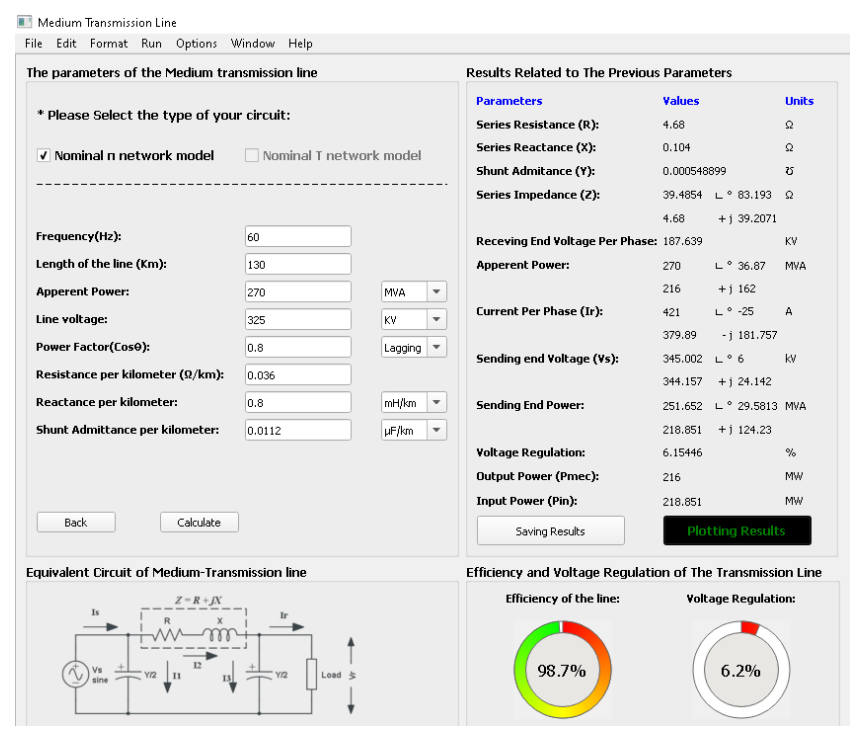

Fig. 10. ITUPSA Middle Transmission Line Parameter Interface

Similarly, the results of the analysis of the parameters for the medium length power transmission line in Figure 9 and the long transmission line in Figure 10 are given. In addition, in the program, optional options such as printing and recording of the results are included.

\section{CONCLUSION}

In this study, a python-based software is designed for electrical power systems. In the study, the software used to support the courses and laboratories given in ITU electrical power engineering department is recommended for students to practice. The program recommended for students for the mathematical parts of the electrical power systems course given in the electrical engineering department in the software was found practical and instructive by the students. The software was written in open code using the Pythonbased PyQt5 module. The software has two language options, there are also plot and favour options. The program is mainly based on object-oriented programming. On the other hand, general information about energy transmission lines including classification methods and mathematical formulas and equivalent circuits have been added to the program without giving details. In the software, a graphical user interfaces (GUI) program, which can make accurate calculations for three different types of energy transmission lines, has been created, and its own custom interface, including line parameters, equivalent circuit, results and waveform, has been created for each type. In this way, the user can make different analyzes by changing the parameter information of the system where a single line diagram is given. By analyzing the results of the analysis numerically, it can compare whether the solutions are correct or not. In addition, it is not necessary to create a new single-line diagram to analyze with different information of the system. In addition, this program has the feature of being integrated into renewable energy systems.

\section{ACKNOWLEDGE}

This study is carried out in the Department of Electrical Engineering of Istanbul Technical University. It was produced from the undergraduate thesis of Yousef Marwan Alkhanafseh under the supervision of T. Cetin Akinci.

\section{Declaration of Conflicting Interests}

The author(s) declared no potential conflicts of interest with respect to the research, authorship, and/or publication of this article.

\section{REFERENCES}

[1] Butler S. The nature of UK electricity transmission and distribution networks in an intermittent renewable and embedded electricity generation future. Imperial College of Science, Technology and Medicine Centre for Environmental Technology. MsC Thesis 2001: 9-12.

[2] Guarnieri, M. "Who Invented the Transformer?". IEEE Industrial Electronics Magazine. 2013, 7 (4): 56-59.

[3] Vaclav S. Creating the Twentieth Century: Technical Innovations of 1867-1914 and Their Lasting Impact, Oxford University Press, 2005, p. 71 Bright A.A. The Electric-Lamp Industry, The Macmillan Company Newyork. 1949. 19-318.

[4] Kiessling F, Nefzger P, Nolasco JF, Kaintzyk U. Overhead power lines. Springer, Berlin, Heidelberg, New York,2003: p. 5

[5] Hughes T.P. Networks of Power: Electrification in Western Society, 1880-1930. Baltimore: Johns Hopkins University Press. 1993: pp. 119-122.

[6] Guarnieri M. The Beginning of Electric Energy Transmission: Part One. IEEE Industrial Electronics Magazine. 2013, 7(1): 57-60.

[7] Guarnieri M. "The Beginning of Electric Energy Transmission: Part Two". IEEE Industrial Electronics Magazine. 2013 7(1): 52-59.

[8] Houston E.J and Kennelly A.E. (1896). The Electric Motor and the Transmission Power. The W. J. Johnston Company. p. 14. Retrieved 2009-01-07.

[9] Engineers, Institution of Electrical,1880. Notes on the Jablochkoff System of Electric Lighting. Journal of the Society of Telegraph Engineers. IX (32): 143. Retrieved 2009-01-07.

[10] Furfari, F. A. Nichols, R. S. The First Electric Power Transmission Line in North America-Oregon City, Oregon. IEEE Industry Applications Magazine.2003: 9 (4): 7-10.

[11] Karady G.G and Heydt G.T. Increasing Student Interest and Comprehension in Power Engineering at the Graduate and Undergraduate Levels. IEEE Transactions on Power Systems. 2000, 11(1):16-21.

[12] Guney I, Kocyigit G and Onat N. Educational software for power system analysis. Int J Electr Eng Educ 2014; 51: 134-145. 
[13] Liserre M, Sauter T and; Hung J.Y. Future Energy Systems: Integrating Renewable Energy Sources into the Smart Power Grid Through Industrial Electronics. IEEE Industrial Electronics Magazine, 2010: 4(1):18-37.

[14] Molina M.G Energy Storage and Power Electronics Technologies: A Strong Combination to Empower the Transformation to the Smart Grid. Proceedings of the IEEE. 2017: 105(11): 2191-2219.

[15] Ali A, Li W, Hussain R. He X, Williams B.W and Memon A.H. Overview of Current Microgrid Policies, Incentives and Barriers in the European Union, United States and China. Sustainability MDPI. 2017, 9(7), 1-28.

[16] A. F. Neyer, F. F. Wu, K. Imhof: 'Object-Oriented Programming For Flexible Software: Example of a Load Flow'; IEEE Transaction on Power Systems, Volume: 5 No: 3, August 1990 pp. 689-696.

[17] A. Abur, F. Magnago, Y Lu: 'Educational Toolbox for Power System Analysis'; IEEE Computer Applications in Power, October 2000, pp. 31-35

[18] Akinci, TC. Applications of Big Data and AI in Electric Power Systems Engineering. In AI and Big Data's Potential for Disruptive Innovation, ed. Moses Strydom and Sheryl Buckley, IGI Global, 2020. 240-260.

[19] J. M. Ngundam, E. R. Ngalemo, F. Kenfack: 'Virtual Lab For Power System Simulation Part 1 Interactive Design Of Power Networks And LoadFlow Analysis'; IEE Power Engineering Journal. 2001;15(4): 211-21.

[20] M. Foley, A. Bose, W. Mitchell, A. Faustini. An Object Based Graphical User Interface for Power Systems'; IEEE Trans. on Power Systems, Volume: 8, Issue: 1, Feb. 1993, Page(s): 97-104.

[21] Working Group on a Common Format For Exchange of Solved Load Flow Data: 'Common Format For Exchange of Solved Load Flow Data'; IEEE Trans. on Power Apparatus and Systems, Vol.PAS-92, No. 6, November/December 1973, pp.1916-1925.

[22] Quinn R.G. Drexel's E4 program: A Different Professional Experience for Engineering Students and Faculty," Journal of Engineering Education, Oct. 1993, pp. 196-202.

[23] Akera A. Setting the Standards for Engineering Education: A History. Proceedings of the IEEE. 2017: 105(9): 1834-1843.

[24] Berry F.C., DiPiazza P.S. and Sauer S.L. The Future of Electrical and Computer Engineering Education. IEEE Transaction on Education. 2003: 46(4):467-476.

[25] Strimel G and Grubbs M.E. Positioning Technology and Engineering Education as a Key Force in STEM Education. Journal of Technology Education. 2016: 27(2): 21-36.

[26] Lewis T. Design and inquiry: Bases for an accommodation between science and technology education in the curriculum? Journal of Research in Science Teaching. 2006: 43(3), 255-281.

[27] Milano F, Vanfretti L and Morataya JC. An open source power system virtual laboratory: the PSAT case and experience. IEEE Transactions on Education, 2008; 51: 17-23.

[28] Yang J and Anderson M. Teaching tool show results through visualization. IEEE Computer Application in Power 1998; 11(1):3742.

[29] Donadel C.B, Fardin J.F and Encarnacao L.F. Educational Tool for Radial Electrical Distribution Networks Analysis and Optimization Studies Involving Distributed Generation Units. International Journal of Electrical Engineering Education. 2018: 55(1):3-13.

[30] Ngundam J. M. Ngalemo E.R and Kenfack F. Virtual Lab For Powe System Simulation Part 1 Interactive Design of Power Networks and Load Flow Analysis. IEEE Power Engineering Journal. 2001: 15(4): 211-215.

\section{BIOGRAPHIES}

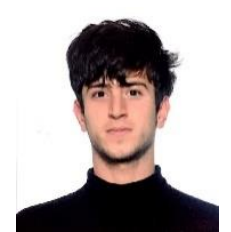

Yousef Marwan Alkhanafseh is currently a software developer, especially a big data engineer, at Turknet Communication Services Company in Istanbul, Turkey. He joined the company as the first big data engineer working on every aspect of data analysis. He has the ability to process tons of terabytes and visualize then in a professional way even on a self-written desktop GUI or on other available data dashboards. Yosef is now, 2021, pursuing master's degree of electrical engineering in Istanbul Technical University, received his B. Sc. degree from the same university and at the same department in 2020 .

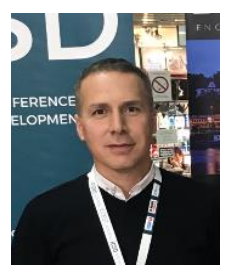

Istanbul, Turkey.
Tahir Cetin Akinci received B.S degrees in Electrical Engineering. M.Sc. and Ph.D. degrees from Marmara University, Istanbul-Turkey. His research interests include Electric power systems, renewable energy, artificial neural networks, deep learning, machine learning, image and signal processing. He has been working as a Professor in Electrical Engineering Department of Istanbul Technical University (ITU) in 